\title{
A New Feature Space for Partial Discharge Signal Separation Based on DWT Coefficient Variance
}

\author{
Maillot Marcos Uriel ${ }^{1}$ and Pessana Franco Martín ${ }^{2}$ \\ 1. Electrical Engineering Department, General Pacheco Regional Faculty, National Technological University, General Pacheco \\ 1617, Argentina \\ 2. Electronic Department, Buenos Aires Regional Faculty, National Technological University, Buenos Aires 1179, Argentina
}

\begin{abstract}
In this paper, a new feature space for PD (partial discharge) signal separation is presented. Three typical PD defects were experimentally reproduced in a laboratory for obtaining independent PD sources. Signals were acquired with a digital storage oscilloscope and then post-processed with DWT (discrete Wavelet transform) for de-noising. The new feature space for PD source separation was constructed with the variance of each Wavelet coefficient vector and was compared with an established feature space for PD source separation; based on the energy of DWT coefficient vectors. After a space reduction by mean of PCA (principal components analysis), the separation capability among them was measured by comparing the final classification error after training a neural network. Results showed that with this new feature space it is possible to separate different sources of PD signals. Later, the feature space proposed was used to separate two PD sources from a real equipment tested. Further analysis on the reduced feature space has shown the band location of PD signals information for separating purpose.
\end{abstract}

Key words: Partial discharges, wavelet transform, singular value decomposition, feature extraction, signal processing.

\section{Introduction}

Partial discharge measurement has become an important role in modern diagnostics technics. As a singular damage mechanism, it requires particular measurement technics capable of detecting such process, which can be invisible to certain kind of measurement no suitable for PD (partial discharge) detecting. The continuous growth on energy quality demanding of the electrical power system had pushed forward the PD measurement technics. Nowadays, in-situ off-line and on-line test of power equipment; on-line monitoring; multi-point and multi-phase acquisitions are some of the standard requirements for PD diagnostics [1]. As defect identification is made by mean of shape analysis of the PRPD (Phase Resolved Partial Discharge) pattern, it is necessary to obtain clean PRPD patterns from noisy environment or

Corresponding author: Marcos Uriel Maillot, electrical engineering, research associate, research fields: partial discharge measurement, condition monitoring of high voltage apparatus, applied signal processing and pattern recognition.
multi-PD sources to make corrects diagnostics [2, 3]. PD signal assessment in time domain with fast acquisition units, capable of recording signal at 500 $\mathrm{MHz}$ (or more) of sampling frequency, had brought the facility to deal with the noisy environment found in electrical power stations by means of digital signal processing $[4,5]$. In addition to noise suppression, the time-resolved PD signal can be used to feature extraction for identifying different sources of PD, a crucial task in assets diagnostics $[2,3,6]$.

In this paper, a new feature space for PD signal separation based on the variance of the DWT (discrete Wavelet transform) coefficients is presented. Three typical PD defects were experimentally reproduced in a laboratory for obtaining independent PD sources. Signals were acquired with a digital storage oscilloscope and post-processed for denoising and features were extracted from its time-domain information. The proposed feature space was compared with a feature space based on the energy of the DWT coefficient for PD source separation $[7,8]$ by 
measurements on the final classification error using a multilayer perceptron Neural Network.

The paper continues with electrode configurations and the experimental setup used to produce the PD defects. Then, a briefly theoretical introduction is presented followed by the data processing procedure. The results found in the artificial defects are firstly analyzed and studied in the results and analysis section; followed by the off-line test made to a real equipment that presented internal PD, where the feature space proposed was tested. Finally the conclusions of both experiences are presented.

\section{Material and Methods}

\subsection{PD sources}

To obtain three well independent sources of PD signals, different electrode configurations were developed. These three classes were:

- Internal in paper-oil system (plate to plate configuration).

- Corona in air (thin metallic electrode in air).

- Corona in oil (needle to plate configuration).

The internal and corona in oil electrodes configuration can be seen in Fig. 1.

To obtain oil degasification and dehumidification, good impregnation and test repeatability in the internal configuration, the procedure described in Ref. [9] was followed.

The corona in air configuration was generated with a wire connected directly upon the coupling capacitor in vertical position to produce a high potential gradient along its length and in its end. In all cases, no other PD source was connected during the test, so individual PD signals were obtained.

\subsection{Equipment, Layout Test and Acquired Data}

The layout is presented in Fig. 2. The test was carried out with the below listed equipment:

- $40 \mathrm{kV}, 2 \mathrm{kVA}$ voltage source.

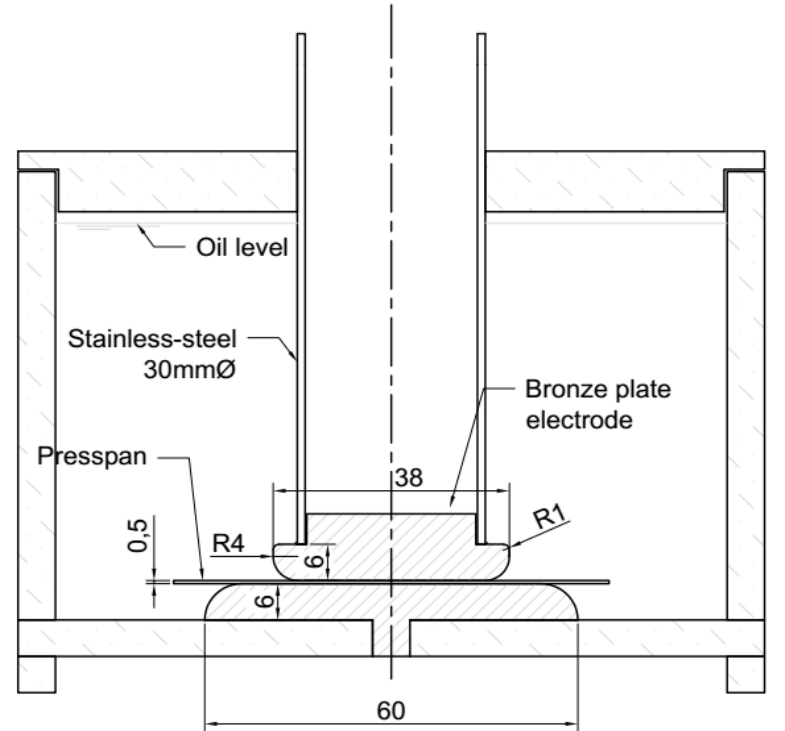

(a) Internal PD.

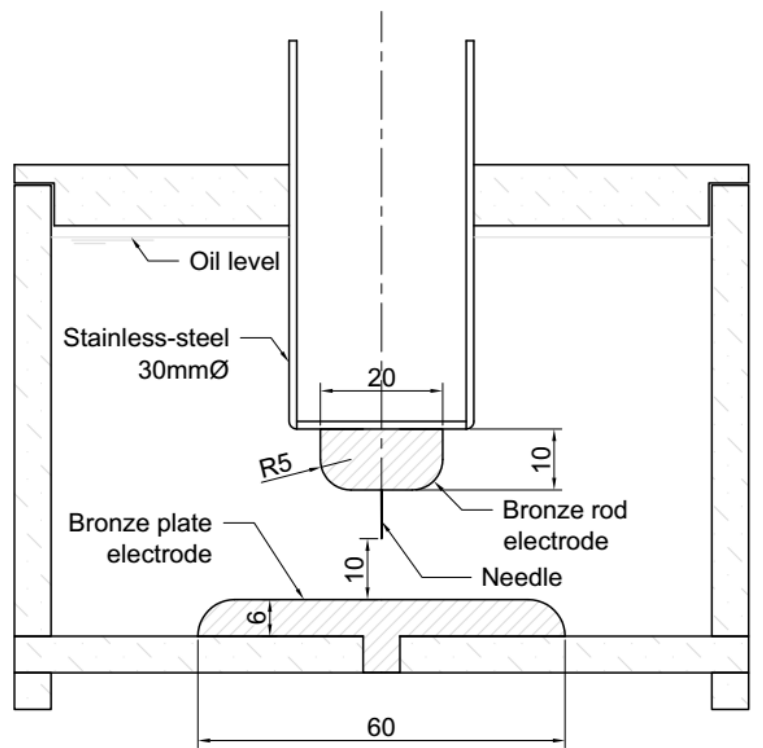

(b) Corona in oil PD.

Fig. 1 Electrodes used in PD configuration. Dimensions are in $\mathbf{m m}$.

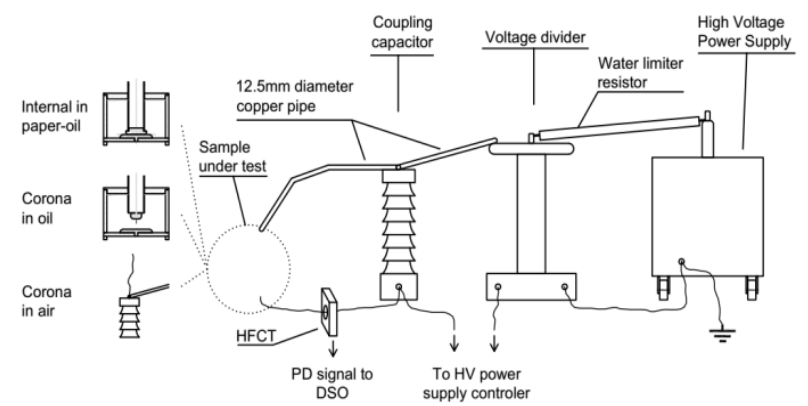

Fig. 2 Layout test. 
- Resistive voltage divider, ratio 10,000:1.

- $17 \mathrm{pF}, 19 \mathrm{kV}$ coupling capacitor.

- 1-80 MHz, $15 \mathrm{mV} / \mathrm{mA} \mathrm{HFCT} \mathrm{clamp.}$

- DSO (digital storage oscilloscope) Agilent DSOX2024A.

To avoid unwanted corona noise in high voltage connections, all these connections were made with a $12.5 \mathrm{~mm}$ diameter copper pipe. The whole test system was submitted at $20 \mathrm{kV}$ without any PD source configuration to ensure no PD activity. Time-resolved PD acquisition configuration was employed to capture PD data; a sampling frequency of $500 \mathrm{MHz}$ was set with a time-domain window of $2 \mu$ s length. Under this acquisition setting, each PD signal was described by a 1000-length data vector. All data collected during tests are summarized in Table 1.

All PD signals were acquired with the DSO under segmented acquisition mode of 250 consecutive pulses as was presented in Ref. [10]. Between each acquisition, the trigger polarity was changed in order to facilitate the capture of both positive and negative pulses. The edge of the trigger was setup in "either" mode. It can be seen from Table 1 that each of the 3 presented classes is composed of 4,000 PD pulses.

Table 1 PD data collected in all measurements.

\begin{tabular}{lllll}
\hline PD Type & Acqn. ID & No. pulses & U test & Trigger lvl. \\
\hline \multirow{4}{*}{ Internal } & int_0 & 1,000 & 15 & $+/-50$ \\
& int_1 & 1,000 & 15 & $+/-75$ \\
& int_2 & 1,000 & 15 & $+/-30$ \\
& int_3 & 1,000 & 15 & $+/-25$ \\
\hline \multirow{4}{*}{ Corona in } & cor_0 & 1,000 & 15 & $+/-65$ \\
air & cor_1 & 1,000 & 6.5 & $+/-15$ \\
& cor_3 & 1,000 & 6.5 & $+/-50$ \\
\hline \multirow{2}{*}{ Corona in } & cor_4 & 1,000 & 19 & $+/-40$ \\
oil & ace_1 & 1,333 & 15 & $+/-40$ \\
& ace_2 & 1,333 & 15 & $+/-100$ \\
\hline
\end{tabular}

PD Type: PD Category.

Acqn. ID: Individual identification of PD acquired set.

No. pulses: Number of PD signals acquired.

$\mathrm{U}$ test: Applied voltage $(\mathrm{kV})$.

Trigger lvl.: Trigger level of acquisition $(\mathrm{mV})$.
The traditional PRPD patterns of each acquired PD class are presented in Fig. 3.

\subsection{Data Processing (Theoretical Introduction)}

The data processing framework of this work was

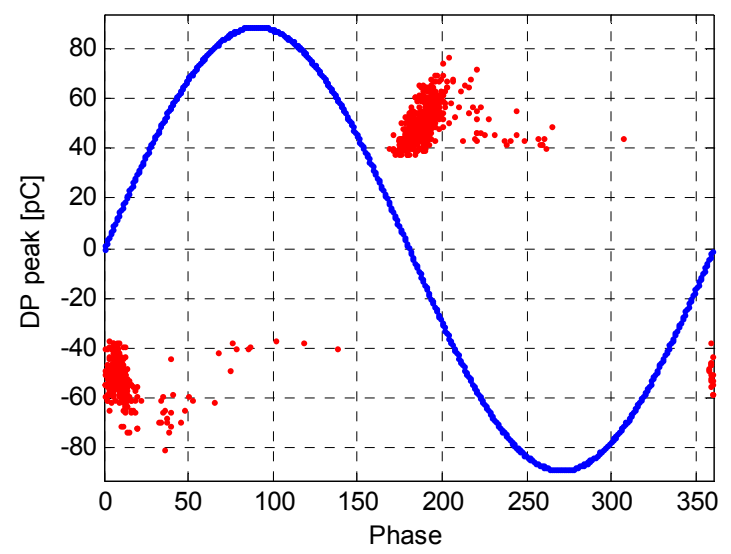

(a) Internal.

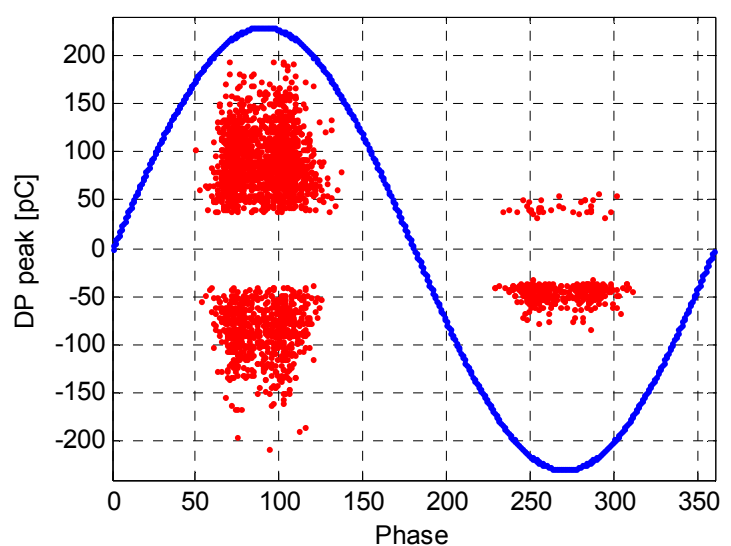

(b) Corona in oil.

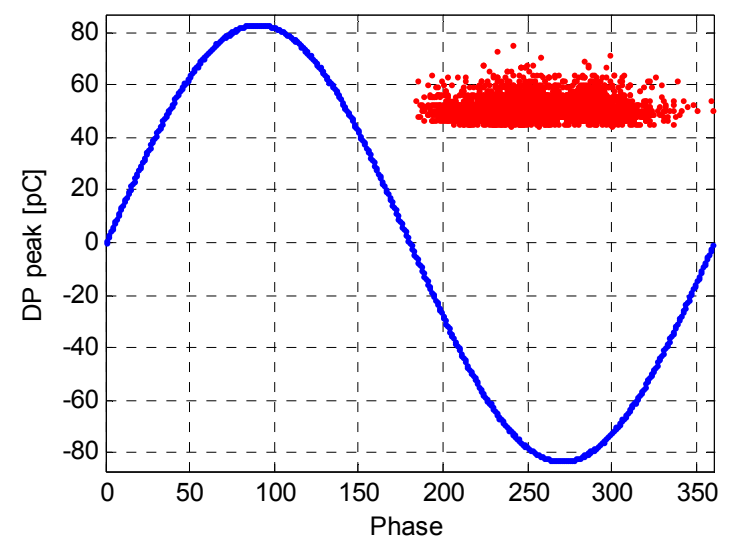

(c) Corona in air.

Fig. 3 PRPD Pattern. 
based on discrete Wavelet transform. The formal fundamentals of Wavelet transform can be found in Refs. [11, 12], and a discrete implementation procedure in Ref. [13]. Many applications of DWT to PD signals (signal denoising and feature space formation) are presented in Refs. [4-6]. Here we will focus on feature space formation of PD pulses.

The DWT projects a signal onto a multidimensional space made of a set of nested sub-spaces. These sub-spaces are defined by a set of basis-functions formed from a "Scale function $\Phi(t)$ " with the two-scale relationship [13]. See Eqs. (1) and (2).

$$
\begin{aligned}
& \phi\left(2^{j} t\right)=\sum_{k} g[k] \cdot \phi\left(2^{j+1} t-k\right) \\
& \psi\left(2^{j} t\right)=\sum_{k} h[k] \cdot \phi\left(2^{j+1} t-k\right)
\end{aligned}
$$

With $j \in \mathrm{Z}<0$, Eqs.(1) and (2) relate through the low pass FIR filter $g[k]$ and the high pass FIR filter $h[k]$ the Scale function in a given level of decomposition with the "Scale function $\Phi(t)$ " and the "Wavelet function $\Psi(t)$ " of the next-lower level respectively. With these recursive formulas, different sub-spaces of the multi-dimensional space are reached, corresponding one level for each $j$. By choosing different Scale function at the beginning of these procedures, different multi-dimensional spaces can be formed.

Similar as in Fourier series analysis, the results of the projections of a signal onto these basis functions are the well-known Wavelet Coefficients. Detail coefficients are related to Wavelet function and the approximation coefficient with the Scale function. In concordance with the beforehand mentioned, they will depend on the Wavelet and Scale function chosen.

The Wavelet Coefficient can be obtained from a bank filter algorithm (see Fig. 4) employed in DWT [13]. This algorithm sheds a vector of coefficient per level of decomposition reached, from whom the time-scale benefits of DWT can be exploited.

As it was mentioned in Ref. [13], each vector of coefficient represents a particular frequency band of the signal, while inside each vector, each element represents at which time of the signal it is located that frequency component. Consequently, we not only separate in different frequency band a certain signal but also, we can study when these frequencies occur by viewing the cadence of such coefficients. This last capability is the support in the present work for PD source separation. Several works have been developed on PD source identification by studying the energy-per-level of the different levels of decomposition reached with DWT $[6,8]$. However, in those methods, the time-scale property of DWT is lost because the energy is totalized for every frequency band (level of decomposition), without studying their distribution. To use the time-scale property, we proposed to analyze the variation of the coefficients in each level and use this magnitude to form a new feature space for PD separation. In this paper, we used the variance of the coefficient in each level to form a feature space for PD pulses separation.

\subsection{Data Processing Procedure}

At first, all PD data were right-side zero-padded to obtain 1,024 points length signals. Then, all PD pulses were decomposed until level 6 with Daubechies 5 (db5) mother Wavelet function. The level of decomposition was selected analyzing the energy reached in the last approximation coefficient vector in comparison with the total energy of the pulse for different level of decomposition. Table 2 shows the results found in this analysis.

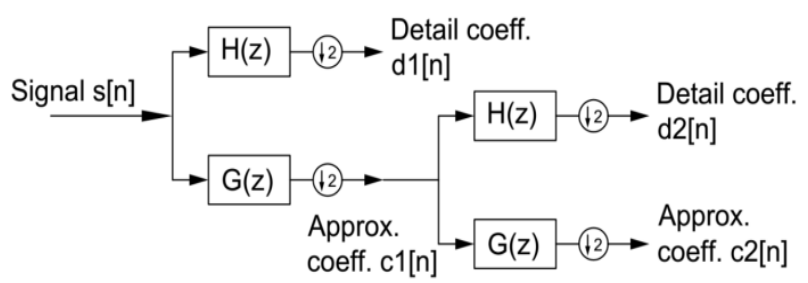

Fig. 4 Two-level filter bank algorithm of DWT. H(z) and G(z) are high pass and low pass finite impulse digital filter respectively, followed by down samplers.

Table 2 Selection of level of decomposition.

\begin{tabular}{ll}
\hline Level & Approximation vector energy \\
\hline 5 & $3.06 \%$ \\
6 & $1.2 \%$ \\
7 & $0.5 \%$ \\
\hline
\end{tabular}


As a first approach, db5 was chosen for testing the proposed method. The relatively short length of its coefficients was considered adequate for this work. For data processing, $d 1$ and $d 2$ coefficients levels were discarded to eliminate high frequency noise (see Fig. 5). The energy $(E(\bullet))$ and variance $(\operatorname{var}(\bullet))$ of each sequence of coefficients was computed. In this way, each PD pulse is now represented by a [1x5] vector of energy-per-Level or a [1x5] vector of variance-per-Level.

Each feature vector found in the above procedure was normalized to allow comparisons of different pulses. The PD energy dependent feature vector $\left(P D f v_{E}\right)$ was normalized by the total energy of the pulse, so each element (dimension) of the feature vector represents a percentage of the total energy of the pulse (see Eq. (3)). In the case of variance, the PD variance dependent feature vector $\left(P D f v_{v a r}\right)$ was normalized against the total variance of the vector [14]. In this case, each dimension of the vector represents a percentage of the vectors total variance (see Eq. (5)).

$$
P D f v_{E}=\left[\frac{E(d 3)}{E t}, \frac{E(d 4)}{E t}, \frac{E(d 5)}{E t}, \frac{E(d 6)}{E t}, \frac{E(c 6)}{E t}\right]
$$

With

$$
\begin{array}{r}
E t=\sqrt{\sum_{n=3}^{6}(d n)^{2}+(c 6)^{2}} \\
P D f v_{\text {var }}=
\end{array}
$$$$
\left[\frac{\operatorname{var}(d 3)}{\text { tot_var }}, \frac{\operatorname{var}(d 4)}{\text { tot_var }_{-}}, \frac{\operatorname{var}(d 5)}{\text { tot_var }}, \frac{\operatorname{var}(d 6)}{\text { tot_var }_{-}}, \frac{\operatorname{var}(c 6)}{\text { tot_var }}\right]
$$

With

$$
\text { tot_var }=\sum_{n=3}^{6} \operatorname{var}(d n)+\operatorname{var}(c 6)
$$

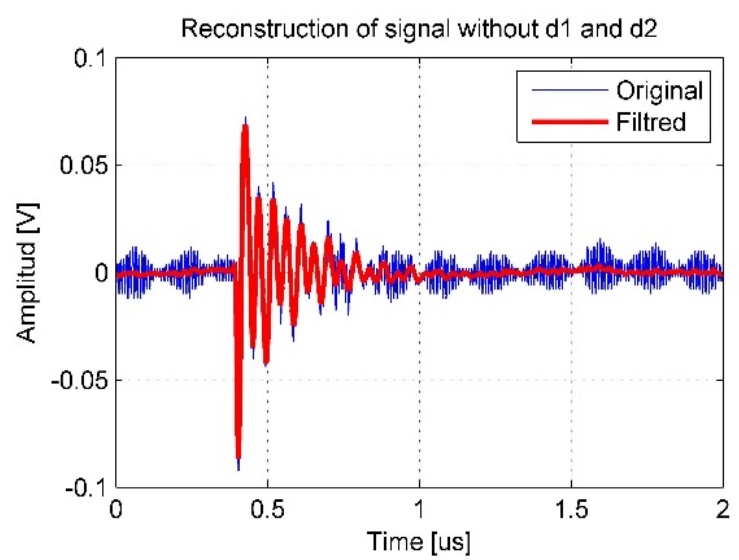

Fig. 5 Original (blue) and filtered signal (red).

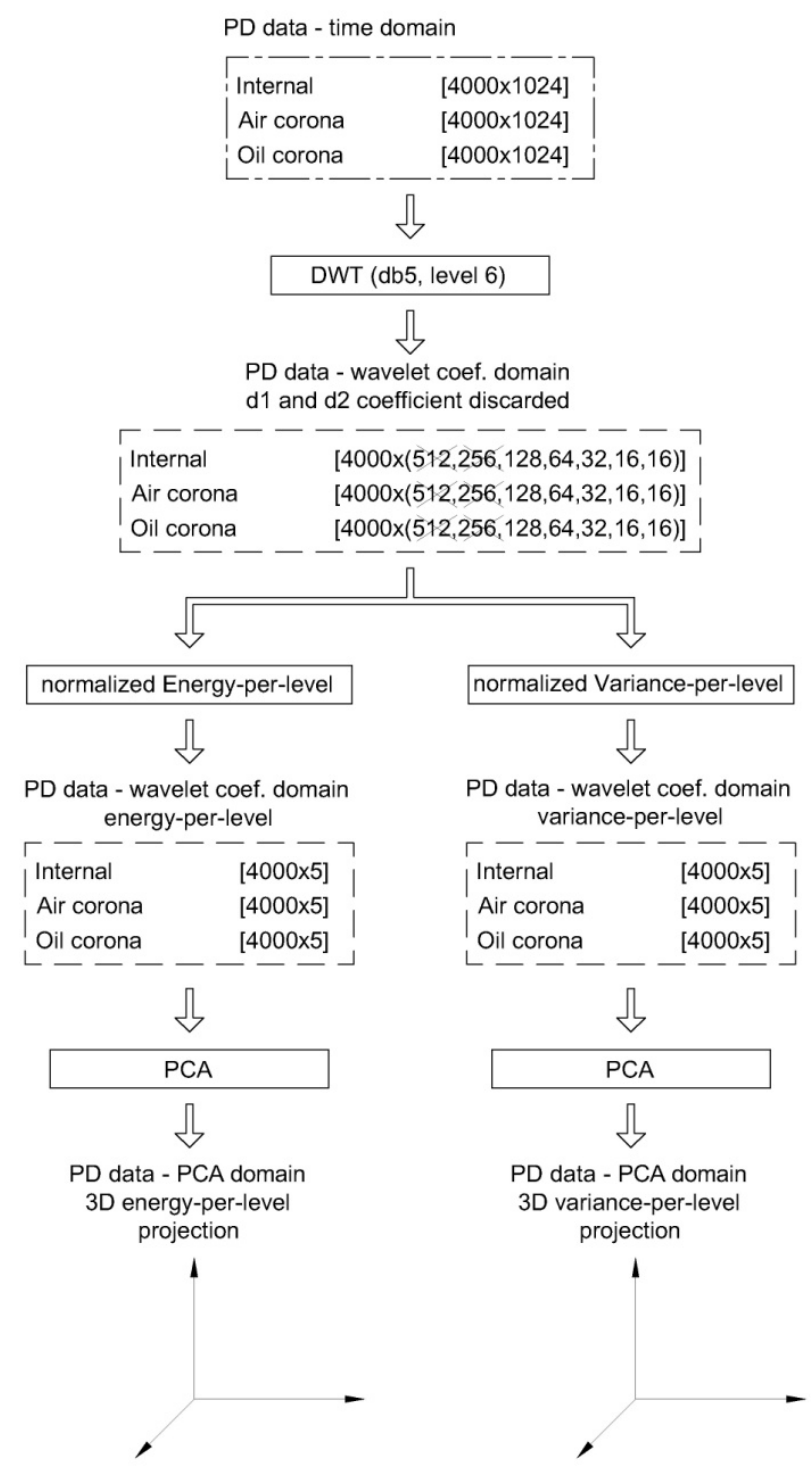

Fig. 6 Flow diagram for data processing.

To have a better visualization of the feature space formed with energy and variance, a space reduction by mean of PCA (principal component analysis) was made $[14,15]$. 3D projections were studied. The flow diagram shown in Fig. 6 exemplifies the performed data processing procedure.

\section{Results and Analyses}

The feature space formed by the first three principal components of energy-per-level and variance-per-level is shown in Figs. 7 and 8 respectively. Different rotations of each axis were used to find the better view of the conformed cloud. 


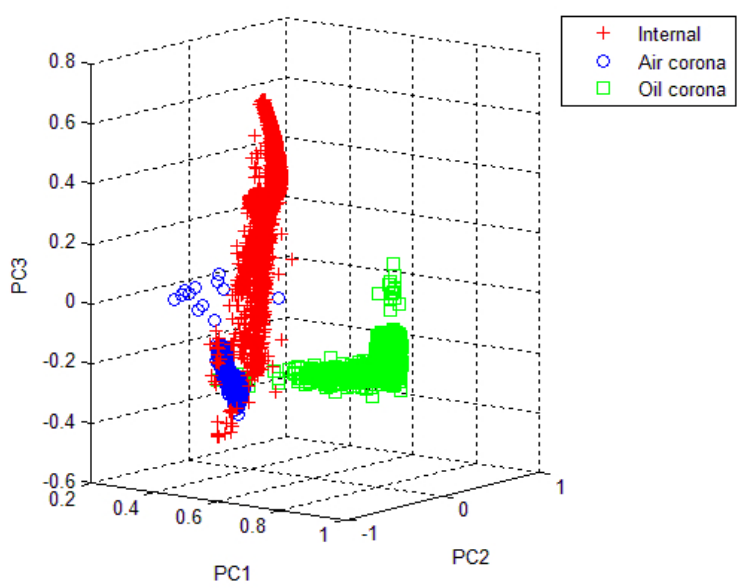

Fig. 7 3D PCA projection of energy per level feature vector.

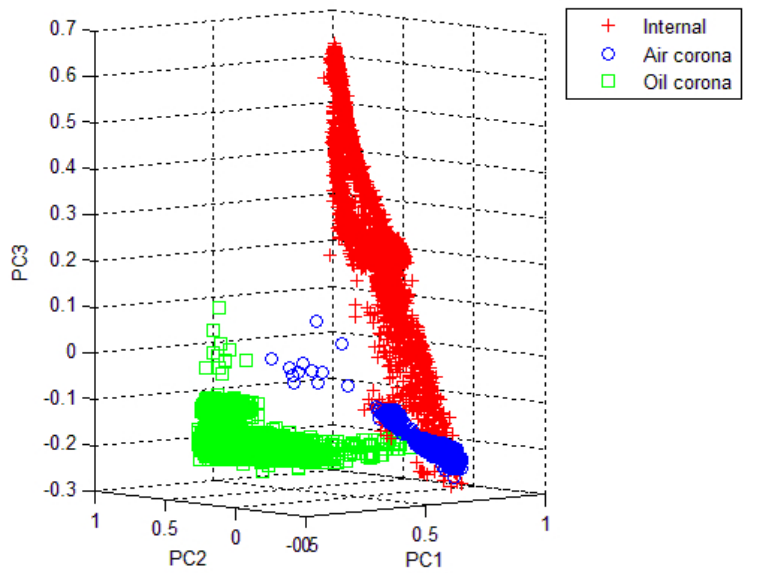

Fig. 8 3D PCA projection of variance per level feature vector.

In a first analysis, both feature spaces can gather together in three different groups, the three target classes. Some apparent superpositions between the clouds are solved by a deep inspection in 2D projections (PC1 vs. PC2; PC1 vs. PC3 and PC2 vs. PC3). Some outliers of each class are the only cases of superposition that were found.

\subsection{Feature Space Comparison}

To have a better comparison, their separation capability was tested with a NN (neural network). To measure this, the classification error of a trained multilayer perceptron NN [16] was employed as a ruler. In Fig. 9, the architecture of the network is presented for both cases, and was fixed (3-10-3), corresponding 3 neurons for the input feature space (the first three principal components), 10 neurons for the hidden layer and 3 neurons for the output (to identify three different PD sources). The network was trained with back-propagation algorithm [16]. The stop criteria were after 50,000 actualization of the network or a total classification error less than $0.8 \%$ (whichever occurs first). Cross-validation with a random chose of $1 / 3$ of the population for training and $2 / 3$ for testing was repeated 20 times in order to obtain a good estimation of the final classification error.

The average MSE (mean square error) and its variance for each feature space after 20 independent training procedures amongst all PD data is presented in Table 3. The results show that both feature spaces were capable of separating the three PD classes.

\subsection{Further Analyses}

A deeper analysis was made studding the result of the PCA projection. The accumulative percentage of total variance explained by each component is presented in Fig. 10 as a function of the number of PCs used.

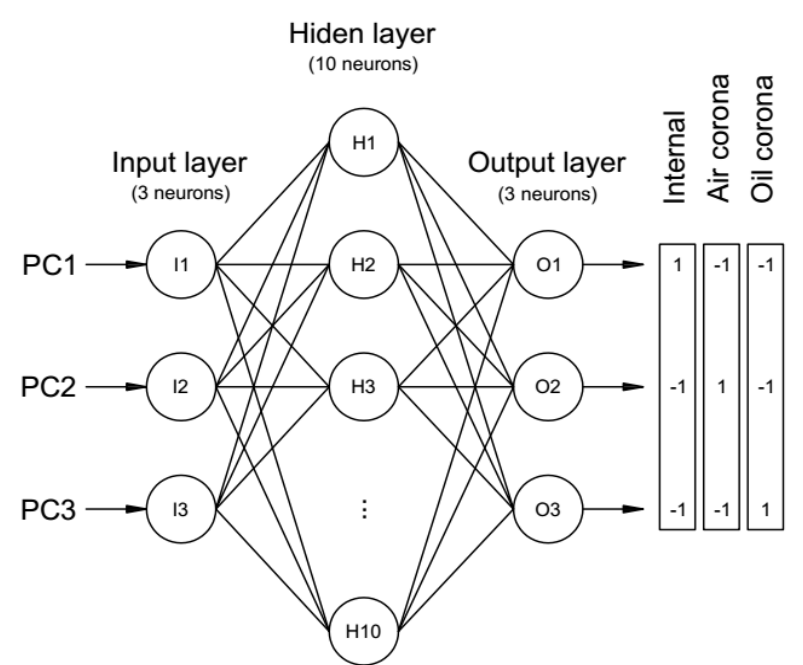

Fig. 9 Neural network architecture.

Table 3 Comparison on classification error.

\begin{tabular}{lll}
\hline Feature space & MSE & Variance \\
\hline Energy dependent & $7.69 \%$ & $2.58 \times 10^{-5}$ \\
Variance dependent & $8.49 \%$ & $2.42 \times 10^{-5}$ \\
\hline
\end{tabular}




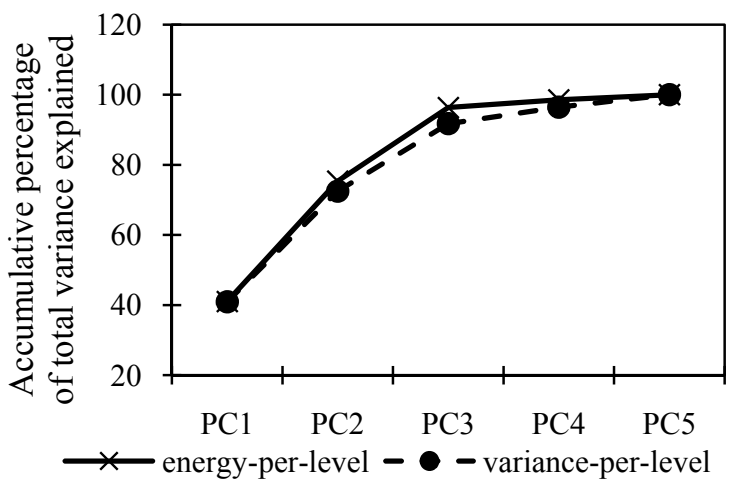

Fig. 10 Accumulative percentage of total variance explained by principal components.

Table 4 Formation of energy-per-level first three PCs.

\begin{tabular}{llll}
\hline Wcfs. & PC1 & PC2 & PC3 \\
\hline $\mathrm{d} 3$ & $37.4 \%$ & $42.5 \%$ & $10.5 \%$ \\
$\mathrm{~d} 4$ & $29.2 \%$ & $17.5 \%$ & $50.5 \%$ \\
$\mathrm{~d} 5$ & $29.5 \%$ & $36.3 \%$ & $36.5 \%$ \\
$\mathrm{~d} 6$ & $2.2 \%$ & $2.3 \%$ & $1.0 \%$ \\
$\mathrm{c} 6$ & $1.7 \%$ & $1.4 \%$ & $1.5 \%$ \\
\hline
\end{tabular}

Wcfs.: DWT vector coefficients.

Table 5 Formation of variance-per-level first three PCs.

\begin{tabular}{llll}
\hline Wcfs. & PC1 & PC2 & PC3 \\
\hline $\mathrm{d} 3$ & $17.9 \%$ & $62.4 \%$ & $17.2 \%$ \\
$\mathrm{~d} 4$ & $24.7 \%$ & $7.3 \%$ & $56.4 \%$ \\
$\mathrm{~d} 5$ & $46.0 \%$ & $28.0 \%$ & $23.7 \%$ \\
$\mathrm{~d} 6$ & $6.2 \%$ & $2.0 \%$ & $1.2 \%$ \\
$\mathrm{c} 6$ & $5.2 \%$ & $0.3 \%$ & $1.5 \%$ \\
\hline
\end{tabular}

Wcfs.: DWT vector coefficients.

It can be seen for both feature vectors, with the first three PCs the $96.2 \%$ and $91 \%$ of the population is represented for energy-per-level and variance-per level respectively. This shows that it is possible to work with a 3-dimensional space, instead of 5 dimensional, simpler graphical arrangements can be done to classify different PD signals. Further studies in the PCAs vectors have shown how each component is formed. Tables 4 and 5 show the relative composition of each component.

For both cases, the $d 3, d 4$ and $d 5$ vectors coefficients are mainly employed to build the principal components, meanwhile $d 6$ and $c 6$ are barely used. This shows that the information located in $d 6$ and $c 6$ frequency band (lowest frequency of the captured PD signal) is not useful for the classification purpose. An exception was observed for $d 6$ and $c 6$ in variance-per-level in PC1, which sums together $11.4 \%$. This finding might be optimized using other Wavelet functions, and it will be studied in future works.

Comparing the energy-per-level feature space vs. the variance-per-level feature space, it can be seen that in the last one, in each principal component, the greatest coefficient vector percentage stands out from the others, meanwhile, in energy-per-level this difference is smaller. This suggests that the variance-per-level feature space, by itself, has more separation capability than energy-per-level, because the projection into PCA generates components that are primarily formed by one of the directions of the original space ( $d 5$ vector coefficient for PC1 and $d 3$ for PC2, for example). This means that in the case of variance-per-level, an acceptable approximation may be done using only $d 5$ and $d 3$ to project the data. In the case of energy-per-level, the projection into PCA generates components that are more equally formed by the directions of the original space ( $d 3, d 4$ and $d 5$ vector coefficients), the same approximation above mentioned will produce worse results.

\subsection{Test on Real Equipment}

To verify the functionality of the feature space under real PD signals, off-line tests on a $33 \mathrm{kV}$ (line to line) oilpaper impregnated current transformer were developed. The equipment, belonging to the local distribution company EDENOR S.A., was withdrawn from service for having internal PD. In Fig. 11 the acquired PRPD pattern is presented where internal PD activity is observed.

The test was carried out in the company's laboratory; a $36 \mathrm{kV}$ PD free voltage source was employed, together with a $45 \mathrm{kV}, 450 \mathrm{pF}, \mathrm{SF}_{6}$ insulated coupling capacitor. The same acquisition system used for the specimens test was employed.

To have an additional PD source in addition to the internal defect, a vertical wire was connected in the 


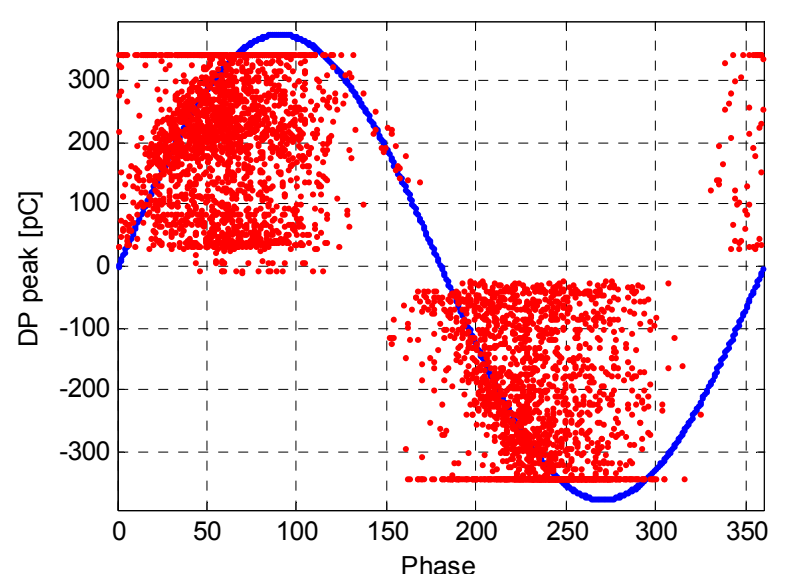

Fig. 11 PRPD Pattern acquired during off-line test on real equipment.

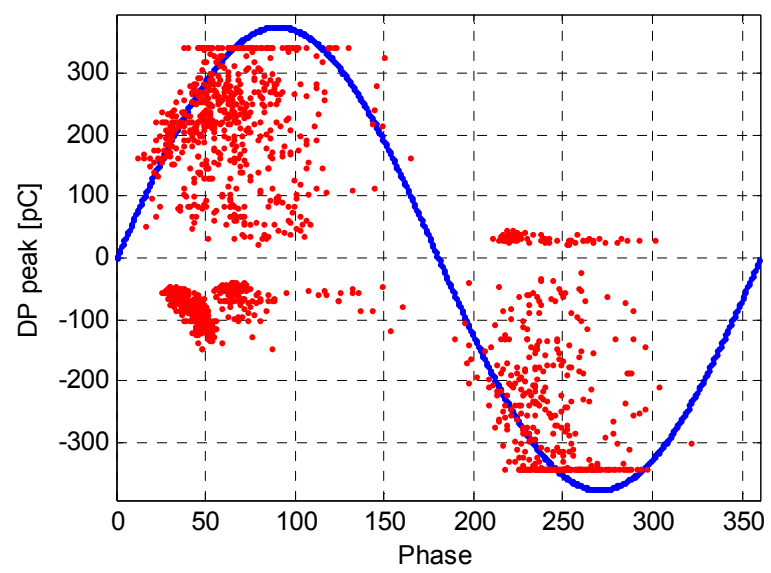

Fig. 12 PRPD Pattern acquired with corona wire.

high voltage connection so it produces corona PD. PRPD pattern acquired with both internal and corona defect is presented in Fig. 12.

To analyze the separate pulses sources, all signals were projected into the feature space proposed in this paper following the same procedure previously presented. In this case, 2D graph of the principal components was enough to plot the data and analyze the clusters. In Fig. 13 the result of the variance dependent feature space of the acquired data is presented with a manual selection of the clusters.

Plotting independently the PRPD pattern of each class (see Fig. 14) the both PD types arise: class 1 (corona defect) and class 2 (internal defect). It can be seen that with the proposed feature space, different sources of PD signals can be differentiated.

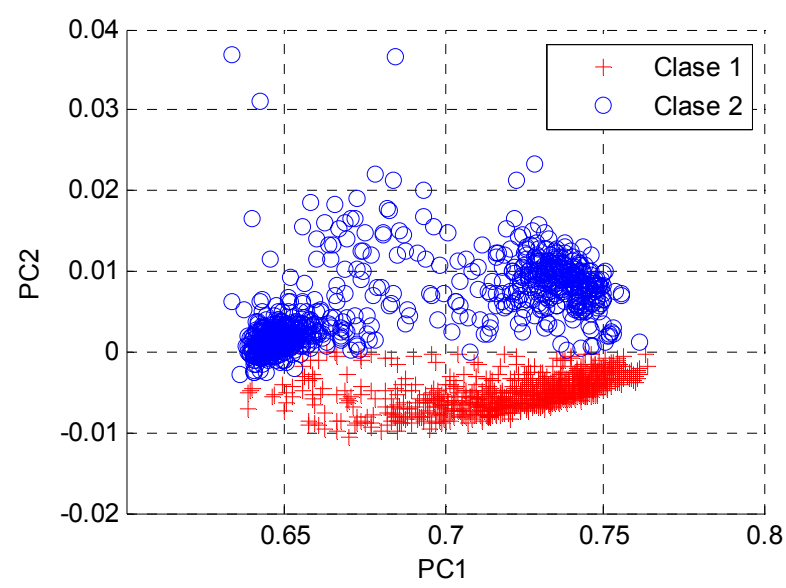

Fig. 13 PD pulses projected onto variance dependent feature space.

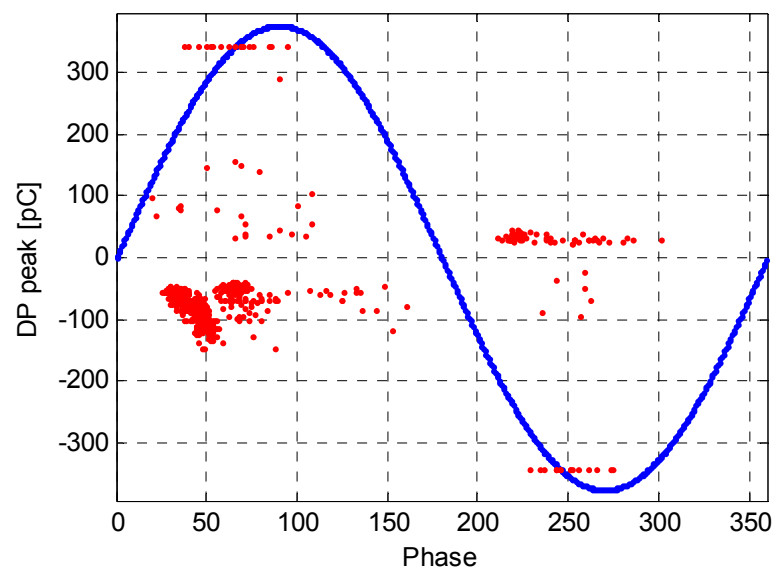

(a) Corona (class 1).

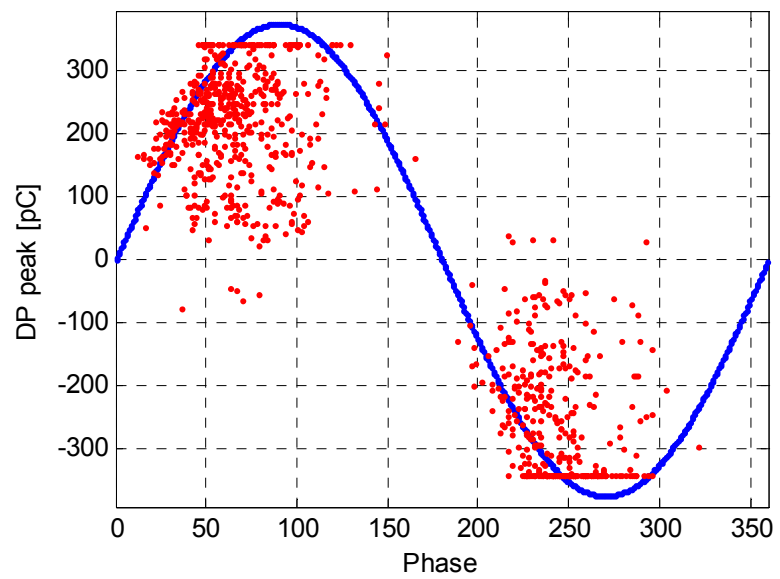

(b) Internal (class 2).

Fig. 14 PRPD Pattern for each of the two classes.

\section{Conclusion and Future Work}

Discrete Wavelet transform to identify different classes of partial discharges was proposed in this paper. 
To exploit the time-scale properties of DWT, the sequence of coefficient inside each level was analyzed by mean of its variance instead of the total energy computation per level. Similar results were found with this new method for feature space formation of time domain PD signals in comparison with the well-known energy-per-level classifier [7, 8].

The main difference among the proposed method and the energy-per-level dependent characterization, is that in the last one, the energy in each frequency band is totalized without studying the distribution of energy in those levels. In the case of variance, it does study the dispersion of the coefficients which may be a closer observation of the physical phenomena under study, because PD pulses are non-stationary signals.

Results found with this methodology show that the variance-per-level is a good feature for characterizing PD pulses, meaning that each of the studied configurations has their own particular energy distribution in each frequency band. This was confirmed for laboratory and real PD signal from off-line test developed on real equipment. On-line tests are pending to be done in order to test the proposed method on an even more realistic environment.

These findings show that not only the PD phenomena have a particular energy allocation in certain band frequency but also it has a particular dispersion of that energy along its occurrence. This particular characteristic might allow separating different sources of PD signal or PD form noise in complex PD patterns, typically found in on-line measurements.

Some limitations of this procedure, are that the PD pulses shape will depend on the transfer function from the PD source to the measurement device, including the PD sensor and connections cables. Different pulse spectrum should be expected between different PD measurement setups. No absolute values of the Principal Component founded here can be extended to all the possible cases. However, this procedure can be used relatively to separate different sources in individual measurement configuration.

Future works under development are the analysis of the feature space proposed with different Wavelet functions, focused on families with different vanishing moments and support to maximize the characterization of PD pulses. In addition to this, others algorithm for dimension reduction, such as LDA (Linear Discriminant Analysis) should be tested and the performance of the processing procedure should be compared with the one presented in this paper.

\section{Acknowledgement}

This research was supported by the "Programas de Becas de Posgrado Cofinanciadas para la formación de Doctores en Areas Prioritarias" from Universidad Tecnológica Nacional Rectorado and Universidad Tecnológica Nacional Regional General Pacheco. Special thanks to Mr. Enciso for the assistance given during the off-line test, the oil samples and the equipment lent for the realization of this work.

\section{References}

[1] Guidelines for Partial Discharge Detection Using Conventional (iec 60270) and Unconventional Methods. 2016. Technical Brochure 662, CIGRE, WG D1.37.

[2] Cavallini, A., Montanari, G. C., Contin, A., and Pulletti, F. 2003. "A New Approach to the Diagnosis of Solid Insulation Systems Based on PD Signal Inference." IEEE Electrical Insulation Magazine 19 (2): 23-30.

[3] Contin, A., Pastore, S., and Paganin, R. 2015. "Evaluation of Spaces for the Separation of Signals due to Multiple PD Sources." In Proceedings of IEEE Electrical Insulation Conference (EIC), Seattle, WA, pp. 209-13.

[4] Machado, R. G. V., and de Oliveira Mota, H. 2015. "A Signal De-noising Technique Based on Wavelets Modulus Maxima Lines and a Self-scalable Grid Classifier." In Proceedings of the IEEE Workshop on Signal Processing Systems (SiPS), Hangzhou, pp. 1-6.

[5] Jayakrishnan, M., Rao, B. N., Meena, K. P., and Arunjothi, R. 2015. "Optimum Threshold Estimator for De-noising Partial Discharge Signal Using Wavelet Transform Technique." In Proceedings of the 2015 International Conference on Condition Assessment Techniques in Electrical Systems (CATCON), Bangalore, pp. 76-82.

[6] Wu, M., Cao, H., Cao, J., Nguyen, H. L., Gomez, J. B., and Krishnaswamy, S. P. 2015. "An Overview of State-of-the-Art Partial Discharge Analysis Techniques 
for Condition Monitoring." IEEE Electrical Insulation Magazine 31 (6): 22-35.

[7] Hao, L., Lewin, P. L., Hunter, J. A., Swaffield, D. J., Contin, A., Walton, C., and Michel, M. 2011. "Discrimination of Multiple PD Sources Using Wavelet Decomposition and Principal Component Analysis." IEEE Transactions on Dielectrics and Electrical Insulation 18 (5): 1702-11.

[8] Xu, J., Niu, H., and Hu, R. 2015. "The Feature Extraction and Pattern Recognition of Partial Discharge Type Using Energy Percentage of Wavelet Packet Coefficients and Support Vector Machines." In Proceedings of the 5th International Conference on Electric Utility Deregulation and Restructuring and Power Technologies (DRPT), Changsha, pp. 1776-9.

[9] Pressboard and press paper for electrical purposes-Part 2: Methods of tests. 2004. Standard, International Electrotechnical Commission.

[10] Maillot, M. 2015. Desarrollo de un equipo de medición de descargas parciales para monitoreo, investigación y diagnostico. Primer Congreso de Investigación y Transferencia Tecnológica en Ingeniería Eléctrica, Buenos Aires, Argentina.

[11] Mallat, S. 2008. A Wavelet Tour of Signal Processing. 2nd Edition. San Diego, CA: Academic Press.

[12] Daubechies. 1991. Ten Lectures on Wavelet. Society for Industrial and Applied Mathematics, Philadelphia, Pennsylvania, Rutgers University.

[13] Goswami, C., and Chan, A. K. 2011. Fundamentals of Wavelets. Theory, Algorithms and Applications, 2nd Edition. Texas A\&M University: John Wiley \& Sons Inc.

[14] Cuadras, M. 2014. Nuevos métodos de análisis multivariante. Madrid, España: CMC Editions.

[15] Nakos, G., and Joyner, D. 1999. Algebra Lineal con Applications. México, U.S. Naval Academy: International Thomson Editores.

[16] Hertz, J. Z., Krogh, A., and Palmer, R. G. 1991. Introduction to the Theory of Neural Computation. Lectures Notes. Volume I. Santa Fe Institute: Addison Wesley Publishing Company, Studies in the Sciences of Complexity. 